\title{
CARACTERIZAÇÃO FíSICO-QUÍMICA E ACEITAÇÃO SENSORIAL DE BEBIDA FUNCIONAL ADICIONADA DE FRUTOLIGOS- SACARÍDEO E FIBRA SOLÚVEL
}

\author{
DANIELA DE GRANDI CASTRO FREITAS * \\ MARISA DE NAZARÉ HOELZ JACKIX **
}

\begin{abstract}
Este trabalho teve como objetivo o desenvolvimento de néctar misto de cenoura e laranja, adicionado de frutoligossacarídeo e pectina cítrica. A influência da adição desses ingredientes funcionais foi avaliada mediante planejamento fatorial completo de suas características físico-químicas e aceitação sensorial. $A$ adição de frutoligossacarídeo em interação com a adição de pectina aumentou o teor de sólidos solúveis e a viscosidade da bebida. O frutoligossacarídeo não acarretou efeito negativo na aceitação sensorial da bebida, mesmo em concentração elevada (15\%). A adição de pectina cítrica como fibra solúvel em concentração acima de $1 \%$ provocou aumento no $\mathrm{pH}$ da bebida e as formulações com essas concentrações não alcançaram boa aceitação pelos julgadores na avaliação sensorial.
\end{abstract}

PALAVRAS-CHAVE: PECTINA; FRUTOLIGOSSACARÍDEO; ALIMENTO FUNCIONAL; ACEITAÇÃO SENSORIAL; SUPERFÍCIE DE RESPOSTA.

\section{INTRODUÇÃO}

A importância do consumo de frutas e vegetais, bem como a possibilidade de usá-los na preparação de alimentos funcionais têm sido revista. HRIBAR e VIDRHI (2001) também relataram o desenvolvimento de bebidas com propriedades funcionais. Há muitos produtos funcionais com diferentes apelos no mercado, cuja eficácia nem sempre está documentada cientificamente (BOGOVIC-MATIJASIC,

* Doutoranda em Tecnologia de Alimentos, Departamento de Tecnologia de Alimentos (DTA), Faculdade de Engenharia de Alimentos (FEA), UNICAMP, Campinas, SP (e-mail: degrandi@fea.unicamp.br/danielafreitas2004@ig.com.br).

** Professora, Doutora em Tecnologia de Alimentos, DTA, FEA, UNICAMP, Campinas, SP. 
2001). Acredita-se que a dieta está relacionada com doenças cardiovasculares, com o câncer, a hipertensão e a obesidade. Os componentes alimentares mais envolvidos com as doenças cardiovasculares e o câncer são as gorduras saturadas e o baixo consumo de fibras, vegetais e frutas (PAS, 2001). Em meados dos anos 80 cresceu no Japão o interesse por alimentos que, além de atender requerimentos sensoriais e nutricionais básicos, exercessem efeitos fisiológicos benéficos (CÂNDIDO e CAMPOS, 1996). ROGELJ (2001) definiu "functional foods", "nutraceuticals" e "designer foods" como alimentos ou ingredientes isolados que trazem benefícios fisiológicos específicos que podem melhorar a saúde.

Os efeitos fisiológicos das fibras dietéticas no metabolismo dos lipídios têm sido amplamente investigados. A maioria dos estudos ressalta as propriedades hipocolesterolêmicas das fibras solúveis. As evidências indicam que diversas fibras solúveis, incluindo guar, pectina, farelo de aveia, fibra de soja e "psyllium" reduzem os níveis de colesterol e glicose no sangue (GRIZARD, DALLE e BARTHOMEUF, 2001; ROY, VEJA-LOPEZ e FERNANDEZ, 2000; DUXBURY, 1993).

Os oligossacarídeos também têm merecido destaque como ingrediente funcional. Várias pesquisas vêm analisando a utilização de oligossacarídeos resistentes (não-digeríveis pelo trato digestivo humano) como fibras. Entre as classes de oligossacarídeos existentes, os frutoligossacarídeos são adicionados aos alimentos porque promovem o crescimento de bactérias bífidas (alimentos probióticos). Por seu efeito antagonista suprimem o crescimento de bactérias putrefativas, balanceando a flora intestinal (alimento prebiótico). Assim, reduzem o acúmulo de metabólitos tóxicos decorrentes de processos fermentativos e a incidência de câncer colônico. O aumento das bifidobactérias também previne a constipação, entre outros efeitos benéficos (TOMOMATSU, 1994; MIZOTA, 1996). Segundo MIZOTA (1996), os frutoligossacarídeos são estáveis ao calor e pH sob as condições normais de processamento de alimentos e para COUSSEMENT (1995) apresentam excelentes propriedades tecnológicas de sabor e textura. Os frutoligossacarídeos (FOS) podem ser simplesmente adicionados (quantidades entre 2 a $5 \%$ são mais comuns) ou usados para substituir outros carboidratos. YUN (1996) afirmou que os FOS são estáveis entre $\mathrm{pH} 4$ e 7 e que sua estabilidade térmica é maior que a da solução de sacarose. Os FOS não são 
degradados durante a maioria dos processos de aquecimento, mas podem perder suas propriedades funcionais quando hidrolisados em frutose em condições muito ácidas ou exposição prolongada a determinados binômios tempo/temperatura (BORNET, 1994; YUN, 1996).

Este trabalho teve como objetivo o desenvolvimento de néctar misto de cenoura e laranja, adicionado de frutoligossacarídeo e pectina cítrica, bem como obter informações sobre suas características físicoquímicas e aceitação sensorial.

\section{MATERIAL E MÉTODOS}

\subsection{MATERIAL}

Foram utilizadas cenouras da variedade Brasília e laranjas da variedade Pêra, adquiridas no comércio da cidade de Campinas (SP-Brasil).

Como ingredientes funcionais foram utilizados: pectina cítrica tipo 8104, alto metoxil-Rapid Set de alto grau de metoxilação (ATM) e esterificação fornecida pela Citrus Colloids S/A (Limeira, Brasil) e frutoligossacarídeo cedido pela empresa ORAFTI Group (Tienen, Bélgica).

\subsection{MÉTODOS}

\subsubsection{Bebida funcional de cenoura e laranja}

Os sucos de cenoura e laranja foram extraídos e misturados na proporção 6:4 (laranja:cenoura). Adoçou-se o suco misto com sacarose na concentração de $6 \%$. A adição de pectina e frutoligossacarídeo foi realizada nas concentrações definidas em delineamento experimental fatorial completo, baseado na Metodologia de Superfície de Resposta. Após a adição, a bebida foi aquecida até $85-90^{\circ} \mathrm{C}$ em equipamento Rotovapor e envasada à quente em garrafas de vidro. Pasteurizou-se a bebida por imersão das garrafas em água em ebulição por 10 minutos, seguida de resfriamento em água corrente. 


\subsubsection{Planejamento Experimental}

Avaliou-se a influência da adição de pectina e de oligofrutose como ingredientes funcionais do néctar mediante planejamento fatorial completo. As variáveis independentes (1) concentração de pectina (PEC) e (2) concentração de frutoligossacarídeo (FOS) resultaram num experimento composto por 11 ensaios. As condições (níveis codificados) estão apresentadas na Tabela 1 e os níveis decodificados de cada variável na Tabela 2.

\section{TABELA1- NÍVEIS CODIFICADOS DAS VARIÁVEIS INDEPENDENTES PARA OS ENSAIOS}

\begin{tabular}{c|cc}
\hline $\mathrm{N}$ do Ensaio & PEC (\%) & FOS $(\%)$ \\
\hline 1 & -1 & -1 \\
2 & +1 & -1 \\
3 & -1 & +1 \\
4 & +1 & +1 \\
5 & 0 & 0 \\
6 & 0 & 0 \\
7 & 0 & 0 \\
8 & $-1,41$ & 0 \\
9 & $+1,41$ & 0 \\
10 & 0 & $-1,41$ \\
11 & 0 & $+1,41$ \\
\hline
\end{tabular}

$\mathrm{PEC}=$ Concentração de pectina .

FOS $=$ Concentração de frutoligossacarídeo.

\subsubsection{Caracterização do suco misto de cenoura e laranja}

A caracterização do suco misto para embasar posteriores avaliações foi feita mediante as determinações de: $\mathrm{pH}$; acidez titulável total, sólidos solúveis ( ${ }^{\circ}$ Brix), teor de polpa, açúcares redutores e totais (AOAC, 1995), teor de pectina (IAL, 1976), viscosidade em Reômetro (Brookfield model DV-III) e vitamina C (ácido ascórbico) conforme BENASSI (1990).

\subsubsection{Propriedades físico-químicas da bebida funcional}

Verificou-se o efeito da adição de pectina e oligofrutose no suco misto pelas seguintes características físico-químicas: $\mathrm{pH}$, acidez titulável 
total, sólidos solúveis ( ${ }^{\circ}$ Brix) (AOAC, 1995), cor em Colorímetro ColorQUEST HunterLab (calibração RSIN, iluminante $\mathrm{D65} / 10^{\circ}$ ) e viscosidade (Reômetro Brookfield modelo DV-III).

\section{TABELA2 - NÍVEIS DECODIFICADOS DAS VARIÁVEIS INDEPENDENTES PARA OS ENSAIOS}

\begin{tabular}{c|cc}
\hline & \multicolumn{2}{|c}{ N veis decodificados } \\
\hline N veis codificados & PEC $(\%)$ & FOS $(\%)$ \\
\hline$-1,41$ & 0,59 & 2,95 \\
-1 & 1,0 & 5,0 \\
0 & 2,0 & 10,0 \\
1 & 3,0 & 15,0 \\
$+1,41$ & 3,41 & 17,5 \\
\hline
\end{tabular}

$\mathrm{PEC}=$ Concentração de pectina .

FOS = Concentração de frutoligossacarídeo.

\subsubsection{Aceitação sensorial da bebida funcional}

Avaliaram-se os atributos sensoriais de aparência, sabor, corpo e impressão global conforme a aceitação do consumidor, mediante escala hedônica não-estruturada de 9 pontos (STONE e SIDEL, 1993). As formulações foram servidas para 22 indivíduos, entre 25 e 40 anos de idade, de ambos os sexos, em três sessões, sendo duas constituídas de 4 e uma de 3 amostras. As amostras foram servidas monadicamente em temperatura de refrigeração doméstica.

\subsubsection{Análise estatística dos dados}

As respostas experimentais foram avaliadas pelo programa Statistica 5.0 (STATSOFT, 1995). A Análise de Variância (ANOVA) e o teste de médias de Tukey ao nível de $5 \%$ de significância $(p \leq 0,05)$ foram efetuados com o auxílio do programa SAS (1993). 


\section{RESULTADOS E DISCUSSÃO}

\subsection{CARACTERIZAÇÃO DO SUCO MISTO DE CENOURA E LARANJA}

Os valores de $\mathrm{pH}$, sólidos solúveis e polpa suspensa do suco misto (Tabela 3) foram compatíveis com os relatados por FREITAS (1999). O valor do $\mathrm{pH}$ enquadrou-se na faixa entre 4,0 a 7,0, considerada de estabilidade para frutoligossacarídeo por YUN (1996). O conteúdo de ácido ascórbico mostrou-se superior ao determinado por FREITAS (1999) $(18,10 \mathrm{mg} / 100 \mathrm{~mL})$.

\section{TABELA 3 - CARACTERÍSTICAS FÍSICO-QUÍMICAS DO SUCO MISTO DE CENOURA E LARANJA}

\begin{tabular}{lc}
\hline Determina I es & Suco Misto de Cenoura e Laranja* \\
\hline $\mathrm{pH}$ & 4,01 \\
$\mathrm{~S}$ lidos soloreis ( Brix) & 8,88 \\
Acidez titul/Æyel (\%) & 0,56 \\
Polpa suspensa (\%v/v) & 7,5 \\
Viscosidade (cP) & 16,18 \\
A oæares redutores (\%) & 2,98 \\
A ocares totais $(\%)$ & 3,28 \\
Pectina (g pectato $/ 100 \mathrm{~mL})$ & 0,21 \\
cido asc rbico $(\mathrm{mg} / 100 \mathrm{~mL})$ & 34,93 \\
\hline
\end{tabular}

* Os dados representam médias de cinco replicatas.

\subsection{PROPRIEDADES FÍSICO-QUÍMICAS DA BEBIDA FUNCIONAL}

As características físico-químicas da bebida funcional estão representadas na Tabela 4. Os efeitos dos fatores concentração de pectina (PEC) e concentração de frutoligossacarídeo (FOS), lineares, quadráticos e da interação entre eles nessas determinações podem ser observados na Tabela 5. Foram construídas as superfícies de respostas e os gráficos de contorno a partir das respostas experimentais dos ensaios (Figura 1), que contribuem para melhor visualização dos efeitos nas características físico-químicas da bebida funcional. 


\section{TABELA 4 - CARACTERÍSTICAS FÍSICO-QUÍMICAS DA BEBIDA FUNCIONAL ADICIONADA DE DIFERENTES CONCENTRAÇÕES DE PECTINA E FRUTOLIGOSSACARÍDEO}

\begin{tabular}{lcccc}
\hline Ensaio & $\begin{array}{c}\text { Acidez Total } \\
(\%)^{*}\end{array}$ & $\mathrm{pH}$ & $\begin{array}{c}\text { S lidos solœeis } \\
\text { ( Brix) }\end{array}$ & $\begin{array}{c}\text { Viscosidade } \\
\text { (cP) }\end{array}$ \\
\hline 1 & 0,59 & 3,82 & 18,43 & 65,5 \\
2 & 0,61 & 3,56 & 18,79 & 528 \\
3 & 0,55 & 3,84 & 21,68 & 70,5 \\
4 & 0,64 & 3,59 & 26,19 & 774 \\
5 & 0,53 & 3,68 & 21,94 & 208 \\
6 & 0,61 & 3,68 & 21,92 & 227 \\
7 & 0,58 & 3,7 & 22,16 & 218 \\
8 & 0,53 & 3,92 & 21,49 & 39,2 \\
9 & 0,68 & 3,55 & 22,8 & 863 \\
10 & 0,62 & 3,67 & 17,29 & 179 \\
11 & 0,57 & 3,72 & 26,80 & 217 \\
\hline
\end{tabular}

* Resultados expressos em mg de ácido cítrico/100 mL da bebida.

TABELA 5 - EFEITO ESTIMADO E GRAU DE SIGNIFICÂNCIA DO MODELO PARA A ACIDEZ, pH, TEOR DE SÓLIDOS SOLÚVEIS E VISCOSIDADE DA BEBIDA FUNCIONAL

\begin{tabular}{|c|c|c|c|c|c|c|c|c|}
\hline & \multicolumn{2}{|c|}{ Acidez } & \multicolumn{2}{|l|}{$\mathrm{pH}$} & \multicolumn{2}{|c|}{ S lidos soloreis } & \multicolumn{2}{|c|}{ Viscosidade } \\
\hline Fatores & $\begin{array}{c}\text { Efeito } \\
\text { Estimado }\end{array}$ & (p) & $\begin{array}{c}\text { Efeito } \\
\text { Estimado }\end{array}$ & (p) & $\begin{array}{c}\text { Efeito } \\
\text { Estimado }\end{array}$ & (p) & $\begin{array}{c}\text { Efeito } \\
\text { Estimado }\end{array}$ & (p) \\
\hline PEC (L) & 0,0806 & 0,1063 & $-0,2586^{\star}$ & 0,0009 & $1,6767^{\star}$ & 0,0031 & $583,6257^{\star}$ & 0,0001 \\
\hline $\operatorname{PEC}(\mathrm{Q})$ & 0,0304 & 0,4684 & $0,0426^{*}$ & 0,0489 & $-0,2248$ & 0,1849 & $251,1673^{\star}$ & 0,0010 \\
\hline FOS (L) & $-0,0209$ & 0,5354 & 0,0298 & 0,0659 & 5,9376 & 2,4530 & $74,4335^{\star}$ & 0,0078 \\
\hline FOS (Q) & 0,0189 & 0,6182 & 0,0021 & 0,8365 & $-0,4166$ & 0,0602 & $-7,9010$ & 0,4100 \\
\hline Pectina ${ }^{\star}$ FOS & 0,035 & 0,4777 & 0,005 & 0,7072 & $2,05^{*}$ & 0,0041 & $120,5^{\star}$ & 0,0061 \\
\hline
\end{tabular}

*Valores significativos ao nível de $95 \%$ de confiança ( $p \leq 0,05)$.

$(\mathrm{L})=$ termo linear.

$(\mathrm{Q})=$ termo quadrático.

$(p)=$ significância estatística.

$\mathrm{PEC}=$ Pectina .

FOS $=$ Frutoligossacarídeo . 


\section{FIGURA 1 - GRÁFICOS DE CONTORNO E SUPERFÍCIES DE RESPOSTA REFERENTES ÀS CARACTERÍSTICAS FÍSICO-QUIIMICAS DA BEBIDA FUNCIONAL}

A

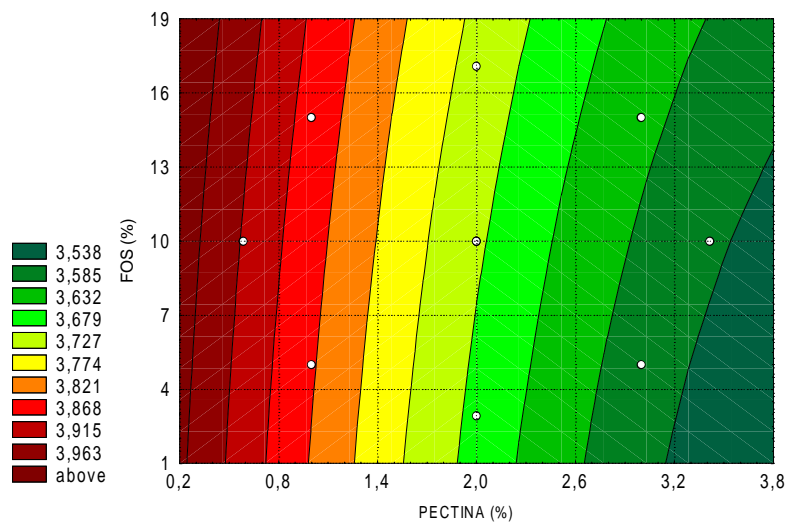

B

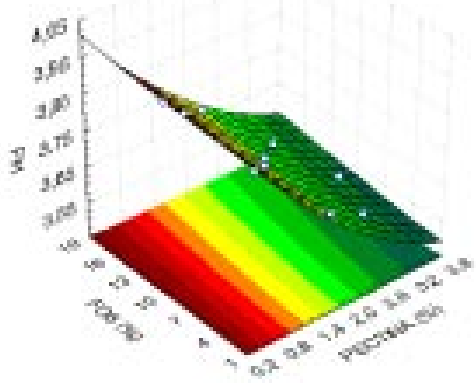

C

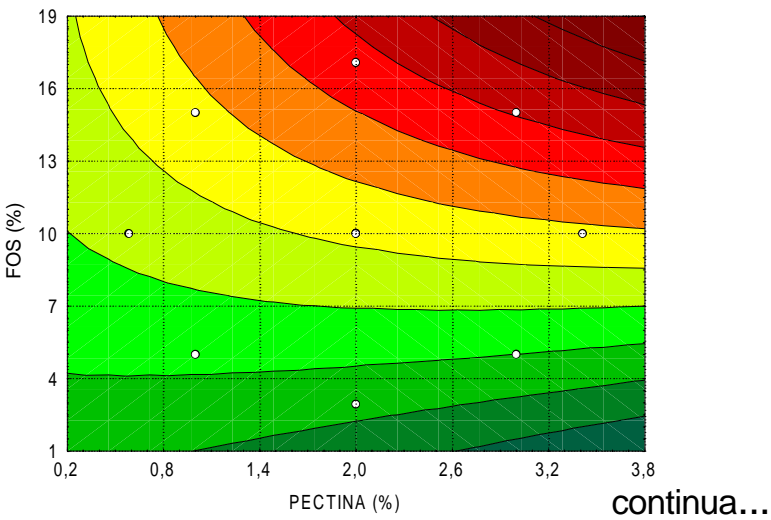




\section{continuação.}

D

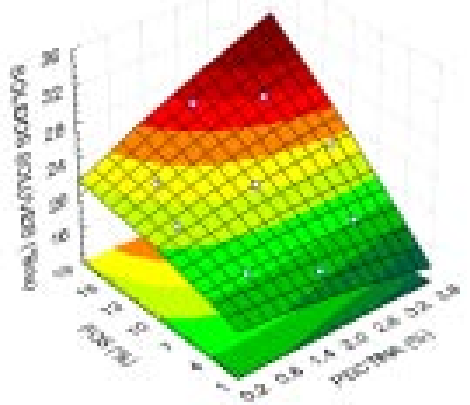

E

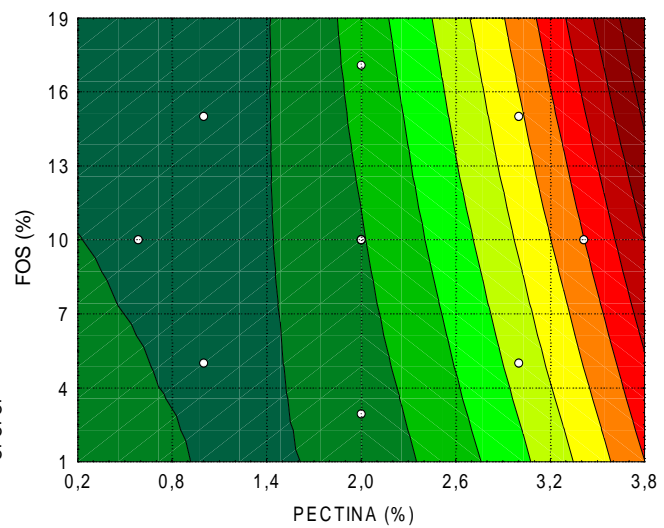

F

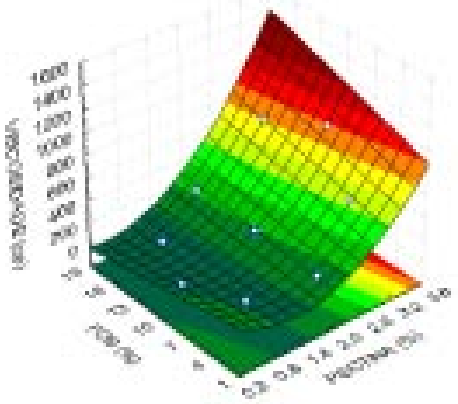

$A=$ Gráfico de contorno referente ao $\mathrm{pH}$ da bebida funcional; $B$ = Superfície de resposta referente ao $\mathrm{pH}$ da bebida funcional;

$\mathrm{C}=$ Gráfico de contorno referente aos sólidos solúveis da bebida funcional;

$D=$ Superfície de resposta referente aos sólidos solúveis da bebida funcional;

$\mathrm{E}=$ Gráfico de contorno referente à viscosidade da bebida funcional;

$\mathrm{F}=$ Superfície de resposta referente à viscosidade da bebida funcional. 
Os valores na coluna dos efeitos (Tabela 5) indicam quanto cada fator influiu no parâmetro estudado. Observou-se que para a acidez total da bebida nenhum dos fatores (concentração de pectina ou de frutoligossacarídeo) exerceu efeito significativo. Já os efeitos dos fatores pectina $L$ e $Q$ foram significativos na variação do $\mathrm{pH}$. O fator pectina $L$, de maior efeito, influenciou negativamente o $\mathrm{pH}$ da bebida, acarretando diminuição no valor de $\mathrm{pH}$ conforme aumento do teor adicionado. Para o teor de sólidos solúveis, os efeitos dos fatores pectina $L$ e interação entre pectina e FOS foram significativos. Quanto maior a concentração dos dois ingredientes na formulação maior o teor de sólidos solúveis. Verificou-se maior efeito da interação que o da concentração de pectina isoladamente. A adição de pectina, predominantemente, e de frutoligossacarídeo contribuiu para o aumento da viscosidade da bebida funcional.

\subsection{ANÁLISE DE COR}

Os produtos obtidos dos ensaios do planejamento experimental foram submetidos à análise instrumental de cor e os resultados estão apresentados na Tabela 6.

\section{TABELA 6 - VALORES MÉDIOS DOS PARÂMETROS DE COR DA BEBIDA FUNCIONAL}

\begin{tabular}{cccccc}
\hline Produto & $\mathrm{L}^{*}$ & $\mathrm{a}^{*}$ & $\mathrm{~b}^{*}$ & O pacidade & D E \\
\hline In natura & $37,56^{\mathrm{f}}$ & $9,42^{\mathrm{f}}$ & $15,49^{\mathrm{de}}$ & 98,07 & - \\
Ensaio 1 & $37,27^{\mathrm{g}}$ & $10,04^{\mathrm{c}}$ & $14,9^{\mathrm{f}}$ & 97,40 & $1,55^{\mathrm{b}}$ \\
Ensaio 2 & $41,76^{\mathrm{a}}$ & $10,75^{\mathrm{a}}$ & $17,33^{\mathrm{a}}$ & 98,48 & $5,17^{\mathrm{h}}$ \\
Ensaio 3 & $35,59^{\mathrm{h}}$ & $8,88^{\mathrm{g}}$ & $13,70^{\mathrm{h}}$ & 97,36 & $4,15^{\mathrm{g}}$ \\
Ensaio 4 & $39,23^{\mathrm{c}}$ & $9,53^{\mathrm{ef}}$ & $15,68^{\mathrm{d}}$ & 98,94 & $1,82^{\mathrm{c}}$ \\
Ensaio 5 & $38,52^{\mathrm{e}}$ & $9,75^{\mathrm{de}}$ & $15,43^{\mathrm{e}}$ & 97,18 & $1,27^{\mathrm{a}}$ \\
Ensaio 6 & $39,43^{\mathrm{c}}$ & $10,42^{\mathrm{b}}$ & $16,24^{\mathrm{c}}$ & 98,28 & $2,41^{\mathrm{d}}$ \\
Ensaio 7 & $38,82^{\mathrm{d}}$ & $9,97^{\mathrm{cd}}$ & $15,63^{\mathrm{de}}$ & 98,16 & $1,49^{\mathrm{a}}$ \\
Ensaio 8 & $35,74^{\mathrm{h}}$ & $9,45^{\mathrm{f}}$ & $14,05^{\mathrm{g}}$ & 97,70 & $3,37^{\mathrm{d}}$ \\
Ensaio 9 & $40,66^{\mathrm{b}}$ & $8,54^{\mathrm{h}}$ & $15,66^{\mathrm{d}}$ & 98,80 & $3,73^{\mathrm{f}}$ \\
Ensaio 10 & $40,51^{\mathrm{b}}$ & $10,57^{\mathrm{ab}}$ & $16,61^{\mathrm{b}}$ & 98,64 & $3,53^{\mathrm{e}}$ \\
Ensaio 11 & $37,65^{\mathrm{f}}$ & $9,51^{\mathrm{ef}}$ & $14,75^{\mathrm{f}}$ & 96,87 & $1,95^{\mathrm{c}}$ \\
\hline
\end{tabular}

*Médias seguidas por letras iguais, na mesma coluna, não diferem significativamente entre si ao nível de 95\% confiança $(p \leq 0,05)$.

$\mathrm{DE}=$ diferença total de cor; $\mathrm{L}=$ luminosidade; $\mathrm{a}=$ cor vermelha; $\mathrm{b}=$ cor amarela. 
A menor diferença total de cor em relação ao suco misto de cenoura e laranja in natura (padrão) foi observada nos ensaios 5 e 7, cujos valores não diferiram estatisticamente ao nível de 5\% de significância. As amostras mais parecidas com o produto in natura foram formuladas com níveis intermediários dos ingredientes pectina e FOS (2 e 10\% respectivamente). Deve-se considerar que a cor do produto sofreu influência do processamento, além dos ingredientes analisados. Todas as amostras mostraram-se opacas, com valores da diferença de luz que atravessa o fundo branco e preto acima de $80 \%$.

\subsection{ACEITAÇÃO SENSORIAL DA BEBIDA FUNCIONAL}

Os resultados da avaliação sensorial dos produtos advindos dos ensaios do planejamento experimental da bebida encontram-se na Tabela 7.

\section{TABELA 7- MÉDIAS DAS NOTAS DOS ATRIBUTOS SENSORIAIS DA BEBIDA FUNCIONAL}

\begin{tabular}{lcccc}
\hline Ensaio & Sabor & Corpo & AparCbcia & Impress $^{\mathrm{a}}$ o Global \\
\hline 1 & $6,91^{\mathrm{ab}}$ & $6,59^{\mathrm{a}}$ & $7,86^{\mathrm{ab}}$ & $6,86^{\mathrm{ab}}$ \\
2 & $4,33^{\mathrm{d}}$ & $3,59^{\mathrm{cde}}$ & $6,27^{\mathrm{ef}}$ & $4,41^{\mathrm{ed}}$ \\
3 & $7,00^{\mathrm{ab}}$ & $6,45^{\mathrm{a}}$ & $7,95^{\mathrm{ab}}$ & $6,63^{\mathrm{abc}}$ \\
4 & $4,68^{\mathrm{cd}}$ & $2,68^{\mathrm{e}}$ & $6,41^{\mathrm{de}}$ & $4,04^{\mathrm{e}}$ \\
5 & $5,45^{\mathrm{cd}}$ & $4,32^{\mathrm{bc}}$ & $7,32^{\mathrm{abc}}$ & $5,31^{\mathrm{cde}}$ \\
6 & $5,41^{\mathrm{cd}}$ & $4,00^{\mathrm{bcd}}$ & $7,00^{\mathrm{cde}}$ & $5,09^{\mathrm{ed}}$ \\
7 & $5,95^{\mathrm{abc}}$ & $4,27^{\mathrm{bc}}$ & $7,22^{\mathrm{abc}}$ & $5,41^{\mathrm{cd}}$ \\
8 & $7,13^{\mathrm{a}}$ & $7,59^{\mathrm{a}}$ & $8,00^{\mathrm{a}}$ & $7,41^{\mathrm{a}}$ \\
9 & $4,36^{\mathrm{d}}$ & $2,81^{\mathrm{de}}$ & $5,54^{\mathrm{f}}$ & $4,54^{\mathrm{de}}$ \\
10 & $5,41^{\mathrm{cd}}$ & $4,95^{\mathrm{b}}$ & $6,91^{\mathrm{cde}}$ & $5,59^{\mathrm{bcd}}$ \\
11 & $5,68^{\mathrm{bcd}}$ & $4,09^{\mathrm{bcd}}$ & $7,32^{\mathrm{bcd}}$ & $5,13^{\mathrm{de}}$ \\
\hline
\end{tabular}

Médias seguidas por letras iguais, na mesma coluna, não diferem significativamente entre si ao nível de $95 \%$ confiança $(p \leq 0,05)$.

Os efeitos dos fatores concentração de pectina (PEC) e concentração de frutoligossacarídeo (FOS) nos atributos sensoriais estudados podem ser observados na Tabela 8. A análise de variância (teste F) evidenciou significância da regressão dos modelos ao nível de 95\% de confiança $(p \leq 0,05)$. Nas Tabelas 9, 10, 11 e 12 encontram-se os valores calculados e tabelados de F. 


\section{TABELA 8- EFEITO ESTIMADO E GRAU DE SIGNIFICÂNCIA DO MODELO PARA OS ATRIBUTOS SENSORIAIS DA BEBIDA FUNCIONAL}

\begin{tabular}{|c|c|c|c|c|c|c|c|c|}
\hline \multicolumn{2}{|c|}{ AparCfacia } & \multicolumn{2}{|c|}{ Sabor } & \multicolumn{2}{|c|}{ Corpo } & \multicolumn{3}{|c|}{ Impress르 o global } \\
\hline Fatores & $\begin{array}{c}\text { Efeito } \\
\text { Estimado }\end{array}$ & (p) & $\begin{array}{c}\text { Efeito } \\
\text { Estimado }\end{array}$ & (p) & $\begin{array}{c}\text { Efeito } \\
\text { Estimado }\end{array}$ & (p) & $\begin{array}{c}\text { Efeito } \\
\text { Estimado }\end{array}$ & (p) \\
\hline PEC (L) & $-1,6545^{\star}$ & 0,0048 & $-2,2004^{*}$ & 0,0092 & $-3,3875^{\star}$ & 0,0012 & $-2,2784^{*}$ & 0,0025 \\
\hline PEC (Q) & $-0,3044$ & 0,1598 & 0,1887 & 0,5369 & $0,9921^{*}$ & 0,0210 & $0,6161^{*}$ & 0,0472 \\
\hline FOS (L) & 0,1505 & 0,3185 & 0,1949 & 0,4516 & $-0,5724^{\star}$ & 0,0413 & $-0,3067$ & 0,1153 \\
\hline FOS (Q) & $-0,340$ & 0,8200 & $-0,0189$ & 0,9447 & 0,3002 & 0,1624 & 0,0092 & 0,9503 \\
\hline Pectina*FOS & 0,0250 & 0,8926 & 0,1150 & 0,7391 & $-0,3850$ & 0,1547 & $-0,0700$ & 0,7105 \\
\hline
\end{tabular}

*Valores significativos ao nível de $95 \%$ de confiança $(p \leq 0,05)$.

$(\mathrm{L})=$ termo linear.

$(\mathrm{Q})=$ termo quadrático.

(p) = significância estatística.

O fator pectina acarretou diminuição na aceitação de todos os atributos sensoriais avaliados pelos julgadores (95\% de confiança). A adição de frutoligossacarídeo exerceu menor influência, interferindo de maneira negativa somente no corpo da bebida.

\section{TABELA 9 - ANÁLISE DE VARIÂNCIA DO MODELO PARA O ATRIBUTOSABOR}

\begin{tabular}{lccccc}
\hline Fonte de Varia ${ }^{\mathrm{a}} \mathrm{o}$ & $\mathrm{SQ}$ & $\mathrm{GL}$ & $\mathrm{MQ}$ & $\mathrm{F}$ calc. & $\mathrm{F}$ tab.** \\
\hline Regress $^{\text {a }} \mathrm{o}$ & 9,6553 & 1 & 9,6553 & $190,14^{*}$ & 5,12 \\
Res duo & 0,4570 & 9 & 0,0507 & & \\
Falta de Ajuste & 0,2760 & 7 & 0,0394 & 0,43 & 19,35 \\
Erro Puro & 0,1810 & 2 & 0,0905 & & \\
Total & 10,1124 & 10 & & & \\
\hline
\end{tabular}

*Valores significativos ao nível de 95\% de confiança ( $p \leq 0,05)$.

${ }^{* *}$ Valores tabelados de $\mathrm{F}$ a $\mathrm{p} \leq 0,05$.

$\mathrm{SQ}=$ Soma Quadrática.

$\mathrm{GL}=$ Graus de Liberdade.

$M Q=$ Média Quadrática. 


\section{TABELA 10 - ANÁLISE DE VARIÂNCIA DO MODELO PARA O ATRIBUTO CORPO}

\begin{tabular}{|c|c|c|c|c|c|}
\hline Fonte de Varia ${ }^{a} 0$ & $S Q$ & $\mathrm{GL}$ & $M Q$ & F calc. & $\mathrm{F} \mathrm{tab.**}$ \\
\hline Regress ${ }^{\mathrm{a}} \mathrm{o}$ & 24,7590 & 3 & 8,2530 & $163,49^{*}$ & 4,35 \\
\hline Res duo & 0,3534 & 7 & 0,0504 & & \\
\hline Falta de Ajuste & 0,2941 & 5 & 0,0588 & 1,98 & 19,30 \\
\hline Erro Puro & 0,0592 & 2 & 0,0296 & & \\
\hline Total & 25,1134 & 10 & & & \\
\hline
\end{tabular}

*Valores significativos ao nível de $95 \%$ de confiança ( $p \leq 0,05)$.

${ }^{* *}$ Valores tabelados de $\mathrm{F}$ a $\mathrm{p} \leq 0,05$.

$S Q$ = Soma Quadrática.

$\mathrm{GL}=$ Graus de Liberdade.

$\mathrm{MQ}=$ Média Quadrática.

TABELA 11 - ANÁLISE DE VARIÂNCIA DO MODELO PARA O ATRIBUTO APARÊNCIA

\begin{tabular}{lccccc}
\hline Fonte de Varia ${ }^{a} \mathrm{o}$ & $\mathrm{SQ}$ & $\mathrm{GL}$ & $\mathrm{MQ}$ & $\mathrm{F}$ calc. & $\mathrm{F}$ tab.** \\
& & & & & \\
\hline Regress $^{\text {a }} 0$ & 5,4589 & 1 & 5,4589 & $141,39^{*}$ & 5,12 \\
Res duo & 0,3474 & 9 & 0,0386 & & \\
Falta de Ajuste & 0,2938 & 7 & 0,0419 & 1,56 & 19,35 \\
Erro Puro & 0,0536 & 2 & 0,0268 & & \\
Total & 5,8064 & 10 & & & \\
\hline
\end{tabular}

*Valores significativos ao nível de $95 \%$ de confiança ( $p \leq 0,05)$.

${ }^{* *}$ Valores tabelados de $\mathrm{F}$ a $\mathrm{p} \leq 0,05$.

$S Q=$ Soma Quadrática.

$\mathrm{GL}=$ Graus de Liberdade .

$M Q=$ Média Quadrática. 


\section{TABELA 12 - ANÁLISE DE VARIÂNCIA DO MODELO PARA O ATRIBUTO IMPRESSÃO GLOBAL}

\begin{tabular}{lccccc}
\hline Fonte de Varia ${ }^{\text {a }}$ o & SQ & GL & MQ & F calc. F tab. ${ }^{* *}$ \\
\hline Regress ${ }^{\text {a }}$ & & & & & \\
Res duo & 10,9333 & 2 & 5,4666 & $99,57^{*}$ & 4,46 \\
Falta de Ajuste & 0,4393 & 8 & 0,0549 & & \\
Erro Puro & 0,3857 & 6 & 0,0642 & 2,39 & 19,33 \\
Total & 0,0536 & 2 & 0,0268 & & \\
\hline
\end{tabular}

*Valores significativos ao nível de 95\% de confiança ( $p \leq 0,05)$.

${ }^{* *}$ Valores tabelados de $\mathrm{F}$ a $\mathrm{p} \leq 0,05$.

$\mathrm{SQ}=$ Soma Quadrática.

$\mathrm{GL}=$ Graus de Liberdade.

$\mathrm{MQ}=$ Média Quadrática.

A partir das respostas experimentais foram construídos os gráficos de contorno e as superfícies de resposta (Figuras 2 e 3), que contribuíram para a determinação das condições de melhor aceitação sensorial da bebida funcional.

\section{FIGURA 2 - GRÁFICOS DE CONTORNO E SUPERFÍCIES DE RESPOSTA REFERENTES À AVALIAÇÃO SENSORIAL DE APARÊNCIA E SABOR DA BEBIDA FUNCIONAL}

A

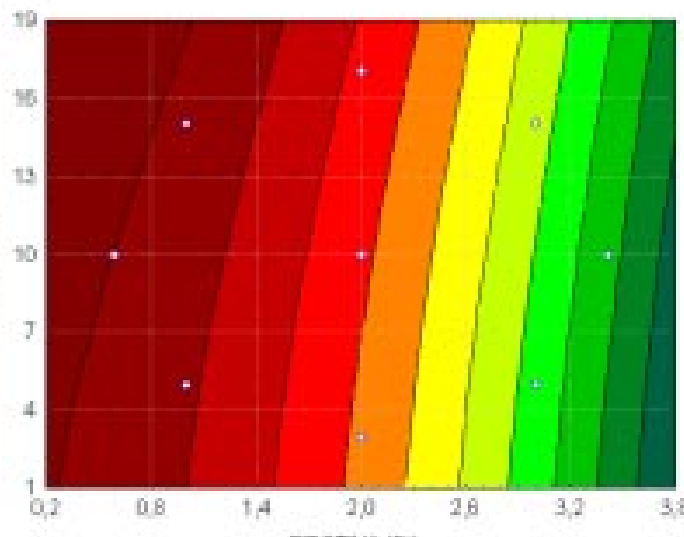

continua... 
continuação.

B

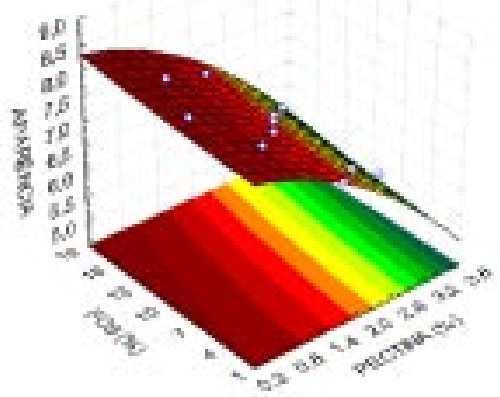

C

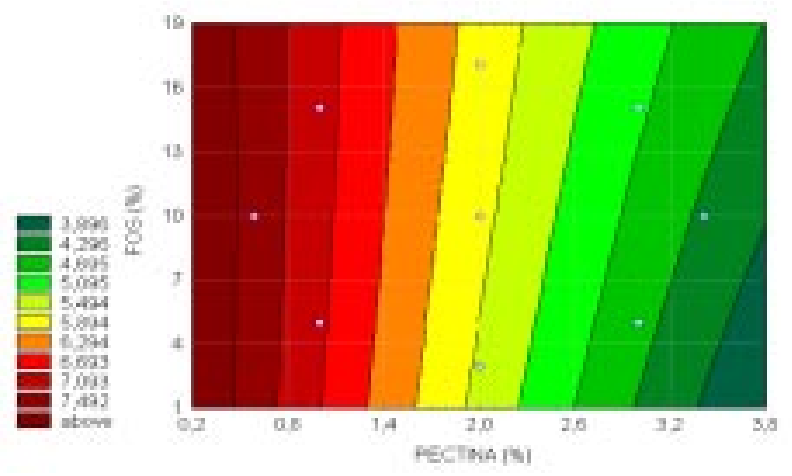

D

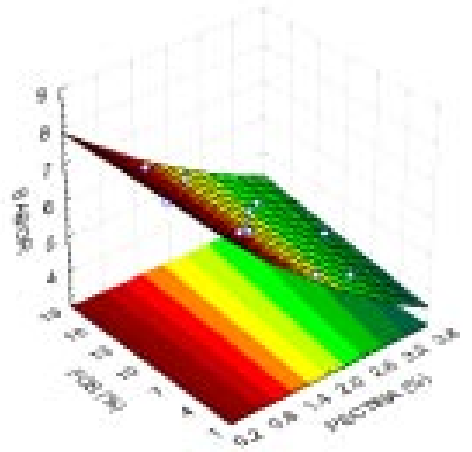

$A=$ Gráfico ae contorno reterente a aparencia aa pebıa tuncıonal;

$\mathrm{B}=$ Superfície de resposta referente à aparência da bebida funcional;

C = Gráfico de contorno referente ao sabor da bebida funcional;

$\mathrm{D}=$ Superfície de resposta referente ao sabor da bebida funcional. 
FIGURA 3 - GRÁFICOS DE CONTORNO E SUPERFÍCIES DE RESPOSTA REFERENTES À AVALIAÇÃO SENSORIAL DE CORPO E IMPRESSÃO GLOBAL DA BEBIDA FUNCIONAL

A

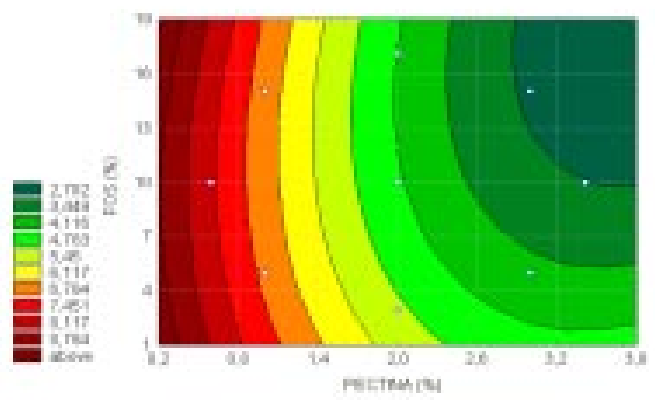

B

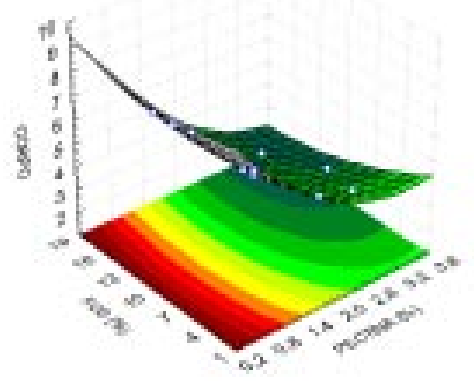

C

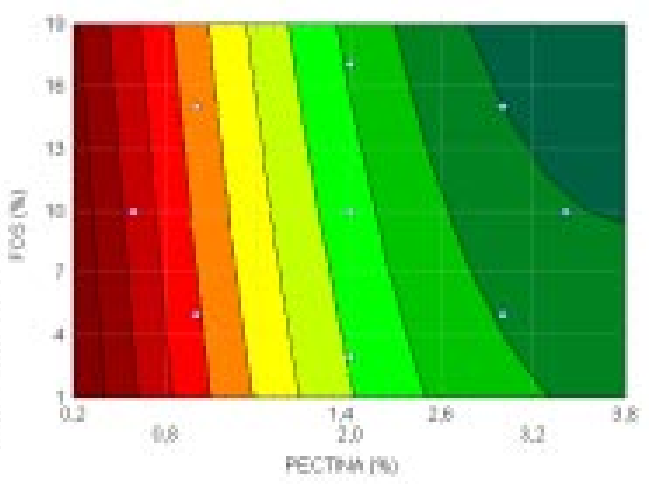

continua... 
continuação.

D

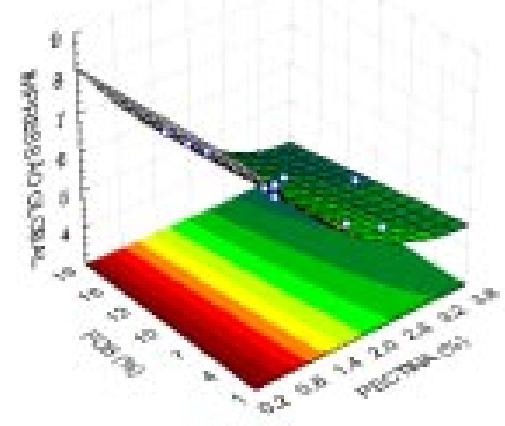

A = Gráfico de contorno referente ao corpo da bebida funcional;

$B$ = Superfície de resposta referente ao corpo da bebida funcional;

$\mathrm{C}=$ Gráfico de contorno referente à impressão global da bebida funcional;

$\mathrm{D}=$ Superfície de resposta referente à impressão global da bebida funcional.

Para a determinação das condições ótimas foram efetuadas sobreposições dos gráficos de contorno (Figuras 2 e 3), obtendo-se a área de máxima resposta (no caso, melhor aceitação sensorial). Assim, a região entre 0,2 e 1,4\% de pectina foi considerada como ótima para a aparência da bebida funcional, podendo-se variar a concentração de FOS entre 1 e 19\%. Outra área, mais restrita, enquadrou-se na região entre 0,2 e $1,1 \%$ de pectina como ótima para o sabor. A faixa entre 0,2 até $0,8 \%$ de pectina representa a região de melhor aceitação quanto ao corpo e impressão global da bebida, podendo-se também variar a concentração de FOS entre 1 e $19 \%$.

\section{CONCLUSÃO}

A adição de frutoligossacarídeo (FOS) como ingrediente funcional em suco misto de cenoura e laranja não acarretou efeito negativo na aceitação sensorial da bebida, mesmo em concentração elevada. As condições ótimas de aceitação enquadraram-se na faixa entre 0,2 e $0,8 \%$ de pectina e 1 a $19 \%$ de FOS.

A adição de frutoligossacarídeo e de pectina aumentou o teor de sólidos 
solúveis e a viscosidade da bebida.

O efeito da adição de pectina cítrica como fibra solúvel em concentração acima de $1 \%$ provocou aumento no $\mathrm{pH}$ da bebida. Além disso, as formulações com essas mesmas concentrações não foram bem aceitas pelos julgadores por conferirem sabor estranho e corpo elevado à bebida funcional.

\section{ABSTRACT}

\section{PHYSICO-CHEMICAL CHARACTERIZATION AND SENSORY ACCEPTANCE OF FUNCTIONAL DRINK ADDED OF FRUCTOLIGOSSACHARIDES AND SOLUBLE FIBER}

This work had as objective the development of a mixed nectar constituted of carrot and orange, added of fructoligossacharides and citric pectin. The influence of the addition of this functional ingredients was evaluated by complete factorial design of their physico-chemical characteristics and sensory acceptance. The addition of fructoligossacharides in interaction with the addition of pectin enhanced the soluble solids rate and the viscosity of the beverage. The fructoligosaccharide didn't cause negative effect on sensory acceptance of the drink, even at high concentration (15\%). The addition of citric pectin as soluble fiber in concentration above $1 \%$ led to an increase in $\mathrm{pH}$ of the drink and the formulations with this concentrations didn't reach good acceptance by the judges in the sensory evaluation.

KEY-WORDS: PECTIN; FRUCTOLIGOSSACHARIDE; FUNCTIONAL FOOD; SENSORY ACCEPTANCE; SURFACE RESPONSE.

\section{REFERÊNCIAS}

1 AOAC. Assoaciation of Official Analytical Chemists. Official methods of analysis of the AOAC International. $16^{\text {th }} \mathrm{ed}$. Arlington, 1995.

BENASSI, M. T. Análise dos estudos de diferentes parâmetros na estabilidade de vitamina $\mathbf{C}$ em vegetais processados. Campinas, 1990. 159 p. Dissertação (Mestrado em Tecnologia de Alimentos) - Faculdade de Engenharia de Alimentos, Universidade Estadual de Campinas.

BOGOVIC-MATIJASIC, B. Assessment of safety and efficacy of functional foods. In: ZLENDER, B.; GASPERLIN, L. (Ed.). Functional foods: proceedings of thematic survey of topic in food science and technology and nutrition for postgraduate studies. Ljubljana (Slovenia): Ljubljana University. Disponível em: <http://server01.bc.unicamp.br/ bases/>. Acesso em: 10 ago. 2003. 
Clinical Nutrition, Paris, v.59, n.3, Suppl, p.763S-769S, 1994.

CÂNDIDO, L. M. B.; CAMPOS, A. M. Alimentos para fins especiais: dietéticos. São Paulo: Varela, 1996. 411 p.

6 COUSSEMENT, P. Pre and symbiotics with inulin and oligofructose. Promising developments in functional foods. Food Technology Europe, v. 2, n. 4, p. 102-104, Dec., 1995.

7 DUXBURY, D. D. Fiber: form follows function. Food Processing, v.54, n.3, p.44-54, Mar., 1993.

8 FREITAS, S. M. L. Utilização de alginato de sódio em texturizados de suco misto de laranja e cenoura de valor calórico reduzido. Campinas, 1999. p. 102. Tese (Mestrado em Tecnologia de Alimentos) - Faculdade de Engenharia de Alimentos, Universidade Estadual de Campinas.

9 GRIZARD, D.; DALLE, M.; BARTHOMEUF, C. Changes in insulin and corticosterone levels may partly mediate the hypolipidemic effect of guar gum and low-molecular weight pectin in rats. Nutritional Researchs, v. 21, n. 8, p. 1185-1190, Aug., 2001.

10 HRIBAR, J.; VIDRIH, R. Fruit, vegetables - functional foods. In: ZLENDER, B.; GASPERLIN, L. (Ed.). Functional foods: proceedings of thematic survey of topic in food science and technology and nutrition for postgraduate studies. Ljubljana (Slovenia): Ljubljana University. Disponível em: <http:// server01.bc.unicamp.br/ bases/>. Acesso em: 10 ago. 2003.

11 IAL. Instituto Adolfo Lutz. Normas analíticas do Instituto Adolfo Lutz: métodos químicos e físicos para análise de alimentos. 2. ed. São Paulo: 1976. $371 \mathrm{p}$.

MIZOTA, T. Functional and Nutritional Foods Containing Bifidogenic Factors. Bulletin of the International Dairy Federation, n 313, p. 31-35, 1996.

13 PAS, M. Minerals. In: ZLENDER, B.; GASPERLIN, L. (Ed.). Functional foods: proceedings of thematic survey of topic in food science and technology and nutrition for postgraduate studies. Ljubljana (Slovenia): Ljubljana University. Disponível em: <http://server01.bc.unicamp.br/ bases/ >. Acesso em: 10 ago. 2003.

14 ROGELJ, I. Sinbiotic milk products: as a case of functional foods. In: ZLENDER, B.; GASPERLIN, L. (Ed.). Functional foods: proceedings of thematic survey of topic in food science and technology and nutrition for postgraduate studies. Ljubljana (Slovenia): Ljubljana University. Disponível em: <http://server01.bc.unicamp.br/ bases/>. Acesso em: 10 ago. 2003.

ROY, S.; VEJA-LOPEZ, S.; FERNANDEZ, M. L. Gender and hormonal status affect the hypolipidemic mechanisms of dietary soluble fiber in guinea pigs. 
Journal of Nutrition, v. 130, n.3, p. 600-607, Mar. 2000.

16 SAS Institute. SAS user's guide: statistics. Cary, USA, 1993.

17 STATSOFT Inc. Statistica for windows [computer program manual]. Tulsa, OK, 1995.

18 STONE, H.; SIDEL, J. L. Sensory evaluation pratics. $2^{\text {nd }}$ ed. San Diego: Academic Press, 1993. 338 p.

19 TOMOMATSU, H. Health effects of oligossacharides. Food Technology, Chicago, v.48, n.10, p. 61-65, Oct., 1994.

20 YUN, J. W. Fructoligossacharides: ocurrence, preparation and application. Enzime and Microbial Technology, n. 19, p. 107-117, 1996. 